Note: This copy is for your personal non-commercial use only. To order presentation-ready copies for distribution to your colleagues or clients, contact us at www.rsna.org/rsnarights.

Ahmed M. Gharib, MB, ChB

Vincent B. Ho, MD

Douglas R. Rosing, MD

Daniel A. Herzka, PhD

Matthias Stuber, PhD

Andrew E. Arai, MD

Roderic I. Pettigrew, PhD, MD
${ }^{1}$ From the Department of Diagnostic Radiology (A.M.G.), National Heart, Lung, and Blood Institute (A.M.G., D.R.R., A.E.A.), and National Institute of Biomedical Imaging and Bioengineering (R.I.P.), National Institutes of Health, Bldg 10, Room 3-5340, MSC 1263, 10 Center Dr, Bethesda, MD 20892; Department of Radiology, Uniformed Services University of the Health Sciences, Bethesda, Md (V.B.H.); Clinical Sites Research Program, Philips Research North America, Bethesda, Md (D.A.H.); and Departments of Radiology, Medicine, and Electrical and Computer Engineering, Johns Hopkins University, Baltimore, Md (M.S.). Received February 8, 2007; revision requested April 11; revision received July 2; accepted August 1; final version accepted September 25. Address correspondence to A.M.G. (e-mail: agharib@nhlbi.nih.gov).

The opinions or assertions contained herein are the private views of the authors and are not to be construed as official or reflecting the views of the Uniformed Services University of the Health Sciences or the Department of Defense.

๑ RSNA, 2008

\section{Coronary Artery Anomalies and} Variants: Technical Feasibility of Assessment with Coronary MR Angiography at $3 \mathrm{~T}^{1}$

The purpose of this study was to prospectively use a whole-heart three-dimensional (3D) coronary magnetic resonance (MR) angiography technique specifically adapted for use at $3 \mathrm{~T}$ and a parallel imaging technique (sensitivity encoding) to evaluate coronary arterial anomalies and variants (CAAV). This HIPAA-compliant study was approved by the local institutional review board, and informed consent was obtained from all participants. Twenty-two participants (11 men, 11 women; age range, 18-62 years) were included. Ten participants were healthy volunteers, whereas 12 participants were patients suspected of having CAAV. Coronary MR angiography was performed with a 3-T MR imager. A 3D free-breathing navigator-gated and vector electrocardiographically-gated segmented k-space gradient-echo sequence with adiabatic T2 preparation pulse and parallel imaging (sensitivity encoding) was used. Wholeheart acquisitions (repetition time msec/echo time msec, $4 / 1.35 ; 20^{\circ}$ flip angle; $1 \times 1 \times 2$-mm acquired voxel size) lasted 10-12 minutes. Mean examination time was 41 minutes \pm 14 (standard deviation). Findings included aneurysms, ectasia, arteriovenous fistulas, and anomalous origins. The 3D whole-heart acquisitions developed for use with $3 \mathrm{~T}$ are feasible for use in the assessment of CAAV.

(0 RSNA, 2008 
oronary arterial anomalies and variants (CAAV) consist of a broad range of disorders, including congenital anomalous origins, aneurysms, ectasia, atresia, and arteriovenous fistulas $(1,2)$, which collectively are found in approximately $0.3 \%-1 \%$ of the population (1-7). However, some types of CAAV can cause chest pain and, in some instances, be life threatening, especially in young individuals (7$9)$. According to the Sudden Death Committee of the American Heart Association, $19 \%$ of sudden deaths in athletes are attributable to CAAV $(1,10)$. Among these anomalies, the more lethal variants are caused by vessels that course between the aortic root and the right ventricular outflow tract or pulmonary artery (11-13).

The use of magnetic resonance (MR) angiography in the evaluation of CAAV has been supported by the American Heart Association (14). The results of several case series in which CAAV has been studied have demonstrated an excellent match between coronary MR angiography and conventional angiography findings at $1.5 \mathrm{~T}(15-20)$. With the growing availability of 3-T MR imaging units, it is important to consider whether higher field strength will confer the potential benefits of higher spatial resolution, faster speed, or both. However, before making this comparison, the pulse sequences that are ordinarily satisfactory at $1.5 \mathrm{~T}$ must be adapted for 3-T imaging because of concerns over the high specific absorption rate, constant magnetic induction field $\left(\mathrm{B}_{0}\right)$ related and radiofrequency magnetic in-

\section{Advances in Knowledge}

- Anomalies and variants of the coronary arteries can be evaluated with three-dimensional (3D) whole-heart acquisitions developed for use at $3 \mathrm{~T}$.

- Sensitivity-encoding accelerated segmented k-space gradient-echo sequence with adiabatic T2 preparation, performed at $3 \mathrm{~T}$, provides sufficient signal-to-noise ratio and image quality with which to assess the coronary arteries. duction field $\left(\mathrm{B}_{1}\right)$-related magnetic field inhomogeneities, motion artifacts, and different relaxation rates resulting in different contrasts at $3 \mathrm{~T}$. It would be useful for the feasibility of 3-T MR imaging in the evaluation of CAAV to be established before the full advantages of higher field strength are explored and exploited. Thus, the purpose of our study was to prospectively use a wholeheart three-dimensional (3D) coronary MR angiography technique specifically adapted for use at $3 \mathrm{~T}$ and a parallel imaging technique (sensitivity encoding) (21) to evaluate CAAV.

\section{Materials and Methods}

One author (M.S.) is compensated as a consultant for Philips Medical Systems (Best, the Netherlands). Another author (D.A.H.) is an employee of Philips Research North America. Philips Medical Systems manufactured the equipment used in our study. All other authors who are not consultants for or employees of Philips Medical Systems had full control over the inclusion of any data or information that might have presented a conflict of interest.

\section{Participants}

Twenty-two participants provided written informed consent for this institutional review board-approved and Health Insurance Portability and Accountability Act-compliant prospective study. All participants underwent imaging within a 11/2-year time span (October 29, 2004, to April 26, 2006). Patients ranged in age from 18 to 62 years; 12 participants were men, and 10 were women. Patients' weights ranged from 56 to $115 \mathrm{~kg}$, and their heart rates ranged from 60 to 90 beats per minute.

Eleven of the 22 participants were symptomatic and were referred for evaluation of possible or known anom-

\section{Implication for Patient Care}

- Clinically diagnostic whole-heart free-breathing 3D images can be obtained in patients and used to evaluate coronary artery anomalies and variants. alous coronary arteries. The other 11 participants were healthy volunteers without prior history of cardiac disease or cardiac-related symptoms. They were recruited from our pool of healthy volunteers on the basis of their availability. There was no prospective attempt at age or sex matching of the volunteers and patients. All volunteers (age range, 18-42 years; mean age, 27.5 years \pm 6.5 [standard deviation]) and patients (age range, 18-62 years; mean age, 42.1 years \pm 15.7 ) were adults. Four healthy volunteers and eight patients were men. A volunteer had an incidental vascular finding on a prior MR image; therefore, this individual was added to the patient pool. Thus, there were 12 patients and 10 healthy volunteers. During this $1 \frac{1}{2}$ year period, three other patients suspected of having CAAV were examined. MR imaging was completed successfully; however, these patients were not included in the patient population because CAAV was not detected with our technique and because no other imaging findings enabled us to confirm the absence of anatomic coronary variations. These three patients were not included in the control group because their pretest probability of having CAAV may have been higher than that of the healthy volunteers.

\section{Published online}

10.1148/radiol.2471070274

Radiology 2008; 247:220-227

\section{Abbreviations:}

CAAV $=$ coronary arterial anomalies and variants

$L A D=$ left anterior descending artery

LCX = left circumflex coronary artery

$\mathrm{RCA}=$ right coronary artery

$3 \mathrm{D}=$ three-dimensiona

\section{Author contributions:}

Guarantor of integrity of entire study, A.M.G.; study concepts/study design or data acquisition or data analysis/ interpretation, all authors; manuscript drafting or manuscript revision for important intellectual content, all authors; manuscript final version approval, all authors; literature research, A.M.G., M.S., A.E.A., R.I.P.; clinical studies, A.M.G., V.B.H., D.R.R.; statistical analysis, A.M.G., A.E.A.; and manuscript editing, all authors

See Materials and Methods for pertinent disclosures. 


\section{Proof of Diagnosis}

We performed conventional angiography prior to MR angiography in eight of 12 patients. Three of these patients also underwent confirmatory coronary computed tomographic (CT) angiography performed with a 16-detector row scanner (MX 8000; Philips, Shelton, Conn). One coronary anomaly was diagnosed initially with echocardiography. The remaining three patients were assessed on the basis of clinical grounds and MR findings. Since the 10 control subjects were healthy adults with no history of heart disease or congenital abnormalities, their pretest likelihood of having CAAV was less than 1\% (1,3$7)$. Thus, they did not undergo other confirmatory imaging because there were no clinical indications to justify subjecting them to additional procedures, radiation, and contrast agents.

\section{MR Angiography}

All 22 participants underwent imaging performed by using a 3-T Intera system (Philips Medical Systems, Best, the Netherlands), a six-element cardiac phasedarray receiver coil, and vector electrocardiographic gating (22). The latter was used to overcome enhanced electrocardiographic changes attributable to the amplified magnetohydrodynamic effect at $3 \mathrm{~T}$. This permits reliable Rwave triggering, even at $3 \mathrm{~T}(23)$. The imaging protocol is a modification of previously described methods at $1.5 \mathrm{~T}$ $(20,24-29)$ and $3 \mathrm{~T}(23,30,31)$.

Scout imaging. - A multisection gradient-echo (repetition time msec/echo time msec, $11 / 2.4 ; 20^{\circ}$ flip angle) scout image was acquired in three orthogonal orientations for (a) volume localization for whole-heart imaging and (b) navigator positioning at the dome of the right hemidiaphragm. This was followed by acquisition of a 3D segmented k-space gradient-echo low-spatial-resolution navigator-gated and vector electrocardiographically gated whole-heart image for localization of the coronary arteries. A two-dimensional selective radiofrequency pulse with 12 revolutions in the $\mathrm{k}$-space and a $15-\mathrm{mm}$ beam radius was used for gating and tracking of respiratory motion (28). The navigator beam was positioned at the dome of the right hemidiaphragm with an 8-mm acceptance window. After the 3D scout image was acquired, a transverse vector electrocardiographically triggered, segmented steady-state free precession cine image series $\left(3.8 / 1.8,45^{\circ}\right.$ flip angle, and 39.6-msec temporal resolution) at the level of the proximal to middle right coronary artery (RCA) was obtained during free breathing. This was done to enable visual determination of the most quiescent period in the cardiac cycle, which was then used to set the trigger delay.

Three-dimensional coronary MR angiography.-After the scout and preparatory examinations were performed, 3D volume-targeted and wholeheart navigator-gated and navigatorcorrected 3D segmented k-space gradient-echo coronary MR angiograms were acquired with this visually identified trigger delay. No intravenous contrast agents were used. Instead, we used a T2 preparation pulse that takes advantage of natural $\mathrm{T} 2$ differences between blood and myocardium (32). However, enhanced $B_{1}$ inhomogeneity at $3 \mathrm{~T}$ limits the ability of this pulse to generate contrast. For this reason, we developed a $B_{1}$ insensitive adiabatic variant of this T2 preparation pulse (33) (50-msec echo time) that incorporates two adiabatic $180^{\circ}$ refocusing pulses and used it to generate contrast between the coronary blood pool and the myocardium. Spectrally selective fat saturation was also used for additional endogenous contrast enhancement between the coronary blood pool and the surrounding tissue. Volumetric shimming was used for 3D acquisitions.

A 3D volume acquisition covering the whole heart $\left(4 / 1.35,20^{\circ}\right.$ flip angle, 55 2-mm-thick partitions interpolated to 1101 -mm-thick partitions during reconstruction) was performed in the sagittal oblique plane, with an acquired voxel size of $1 \times 1 \times 2 \mathrm{~mm}$. To shorten imaging time, a transaxial orientation was used in two patients with a horizontal heart. This 3D whole-heart imaging technique was implemented in all 22 participants. Because to our knowledge this technique was not implemented at 3
$\mathrm{T}$ in patients before our study and to ensure the diagnostic value of the images in the first 10 patients, additional 3D volume-targeted coronary MR angiography was performed. This smaller volume was oriented parallel to the major axes of the left and right coronary arterial system off the transaxial and/or oblique sagittal planes $\left(8 / 2.1, \alpha=20^{\circ}\right.$, 10 3-mm-thick partitions interpolated to 201.5 -mm-thick partitions during reconstruction). A $0.7 \times 1 \times 3$-mm voxel was obtained for this $3 \mathrm{D}$ volume-targeted acquisition. Real-time navigator technology (5-mm gating window, section tracking) (34) was used for respiratory motion suppression for the $3 \mathrm{D}$ whole-heart and 3D volume-targeted sequences. The acquisition window for both 3D segmented gradient-echo sequences was approximately $75 \mathrm{msec}$. Parallel imaging (sensitivity-encoding acceleration factor of two) (21) was used when the acquisition time of either of these 3D volumes exceeded 7 minutes. For all sequences, repetition times and flip angles were set to operate within the specific absorption rate limits set by the manufacturer. For all participants, the radiologist overseeing the study (A.M.G.) recorded the success rate for completing the $3 \mathrm{D}$ wholeheart acquisitions and the duration, total imaging time, and respiratory navigator efficiency of these examinations.

\section{Image Postprocessing and Analysis}

Curved multiplanar reformation was performed with the SoapBubble Tool (Philips Medical Systems) (35), commercially available image analysis software (Virtual Place Advance; Aze, Tokyo, Japan), or both. The Virtual Place Advance software was also used to generate 3D volume-rendered images. Images obtained in all 22 participants were randomly reviewed in consensus by two radiologists (A.M.G., V.B.H.) who were blinded to the type, location, and presence of CAAV detected previously with other imaging modalities. The radiologists had 5 (A.M.G.) and 15 years (V.B.H.) of experience in the interpretation of cardiac and coronary MR images. 
The two radiologists looked for CAAV and measured the diameters of the dilated coronary vessels. The maximum cross-sectional diameter was measured on either the source images or the curved multiplanar reformatted images. Where the diameter was measured depended on the orientation of the dilated vessel. MR angiographic measurements were obtained with a measuring tool available on the aforementioned commercially available imaging analysis software. Measurements on the conventional angiograms were obtained by a cardiologist (D.R.R., 35 years of experience). Calibration was achieved by comparing vessel measurements with the known diameter of the angiographic catheter on the same image.

\section{Statistical Analysis}

Linear regression analysis was performed to determine the relationship and correlation between the diameters of the dilated coronary vessels obtained with MR angiography and those obtained with conventional angiography. The mean diameters and standard deviations obtained with the two imaging modalities were calculated. MedCalc for Windows (version 8.1.1.0; MedCalc

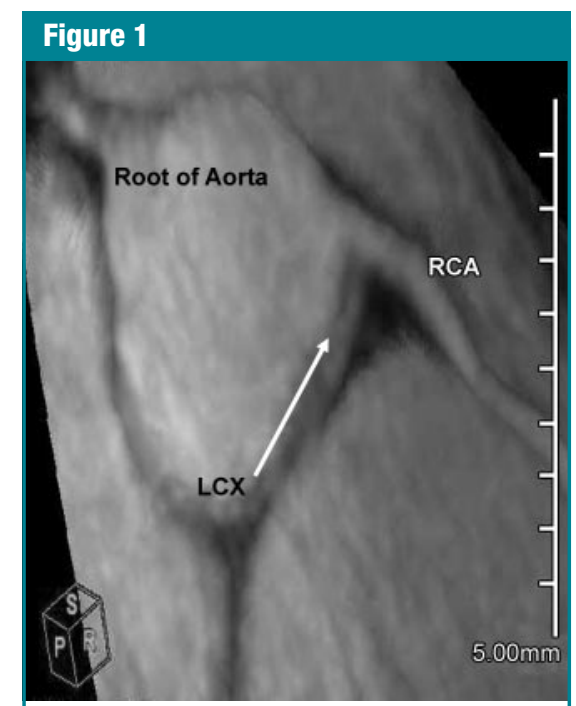

Figure 1: Maximum intensity projection image in the right posterior oblique view shows common origin of the LCX and the RCA from the right sinus of Valsalva in a 42-year-old man with chest pain and heart palpitations.
Software, Mariakerke, Belgium) was used for statistical analysis, and Excel 2003 (Microsoft, Redmond, Wash) was used to generate a scatterplot. $P \leq .05$ was considered to indicate a significant difference.

\section{Results}

\section{Whole-Heart Acquisition Success Rate}

Whole-heart MR angiograms were obtained successfully in 10 of 12 patients and in all 10 healthy volunteers. Successful whole-heart angiograms were not obtained in two patients: In one patient, ventricular bigeminy was seen on electrocardiograms and resulted in poor image quality. The other patient experienced diaphoresis and restlessness, requiring early termination of the examination. To shorten the examination time in these two patients, smaller targeted transverse and oblique volumes that focused around the aortic root in the first patient and around the right atrioventricular groove in the second patient were obtained.

\section{Imaging Duration}

The whole-heart imaging technique had a navigator efficiency of $50 \%-60 \%$ and was used to successfully acquire images in 10-12 minutes. The mean total examination time (including time needed to obtain scout, coronary localizer, cine, and reference images for sensitivity encoding and for the large and small 3D volumes) was 41 minutes \pm 14 . Examination time depended on the participant's heart rate and the navigator efficiency.

\section{Comparison of MR Findings with Proof of Diagnosis}

MR diagnosis was possible in the 10 patients in whom whole-heart imaging was successfully performed, as well as in the remaining two patients who underwent targeted 3D imaging. Nine patients had findings that were correctly identified when compared with images obtained with the available reference standard (conventional coronary angiography, $n=8$; transthoracic echocardiography, $n=1$ ). In the patient who underwent transthoracic echocardiography, further evaluation and radiation exposure were unnecessary given the coinciding anatomic findings at both MR angiography and echocardiography. In the three patients who did not undergo a confirmatory examination, coronary MR angiography revealed a variant in coronary artery origin that did not have any clinical implications. Further radiographic angiographic evaluation was deemed inappropriate in these patients because of the low risk indicated by the findings. The 10 asymptomatic healthy volunteers had no evidence of CAAV at MR imaging.

\section{MR Findings and Analysis}

All 12 patients had CAAV. Abnormalities included anomalous origin of the circumflex coronary artery $(n=3)$; coronary artery aneurysms $(n=3)$, including ectatic vessels; arteriovenous fistula $(n=1)$; common origin of the left anterior descending artery (LAD) and the RCA $(n=1)$; high origin of the left main coronary artery and the RCA $(n=3)$; and aortic origin of the conus branch $(n=1)$.

Anomalous vessel origin or vessel origin variants. - Three patients had an anomalous left circumflex coronary artery (LCX) that originated from the right sinus (Fig 1) and coursed between the root of the aorta and the left atrium before it returned to its usual position in the left atrioventricular groove. MR findings coincided with prior imaging findings (conventional angiography, $n=$ 2; echocardiography, $n=1$ ).

Another patient had an anomalous LAD that originated from the right sinus of Valsalva. The LAD passed anteriorly to the pulmonary artery before returning to its usual course in the anterior interventricular groove (Fig 2); these findings corresponded to the conventional angiography findings.

In three other patients, the left main coronary artery and the RCA originated from the ascending aorta or from a site that was higher than usual $(1,2)$. A conus branch coronary artery that originated from the aorta instead of from the RCA was identified in one participant who was originally in the volunteer 
group but was added to the patient pool (Fig 3). Although this is a small branch, it was clearly seen with coronary MR angiography. Three of the patients with minor CAAV did not undergo confirmatory studies.

Arteriovenous fistula.-A congenital arteriovenous fistula between the LCX and the right ventricular apex (Fig 4) was seen in one patient. This finding was seen initially on a conventional angiogram when the patient was a child, and it was confirmed with coronary CT angiography findings obtained a few days after coronary MR angiography.

Dilated vessels. - In the following patients, the measurements on MR angiograms were compared with the measurements on conventional angiograms: A 48-year-old female patient had aneurysmal dilatation of the left main coronary artery and an ectatic proximal LAD (Fig 5). Two other patients with coronary aneurysms had clinical findings that were consistent with Job syndrome (hyperimmunoglobulin E syndrome), which is associated with hereditary connective tissue abnormalities (36). One of these patients had (a) aneurysms in the left main coronary artery, middle LAD, and second diagonal branch and $(b)$ an ectatic proximal and middle RCA. The second patient with Job syndrome had less severe findings, with a small aneurysm in the proximal LAD and the ectatic proximal RCA. The MR findings in these three patients closely matched the conventional coronary angiography findings.

\section{Analysis of Dilated Coronary Vessel Measurements}

A total of seven dilated coronary vessels in three patients were measured on conventional and MR angiograms. The mean diameter of the dilated coronary vessel was $7.5 \mathrm{~mm} \pm 2.2$ on MR angiograms and $6.3 \mathrm{~mm} \pm 2.2$ on conventional angiograms. A scatterplot of these measurements (Fig 6) shows the close relationship between the MR angiography measurements and the conventional angiography measurements. Correlation coefficients $\left(r=0.97, R^{2}=0.95\right)$ enabled us to confirm this close correla-

\section{Figure 2}

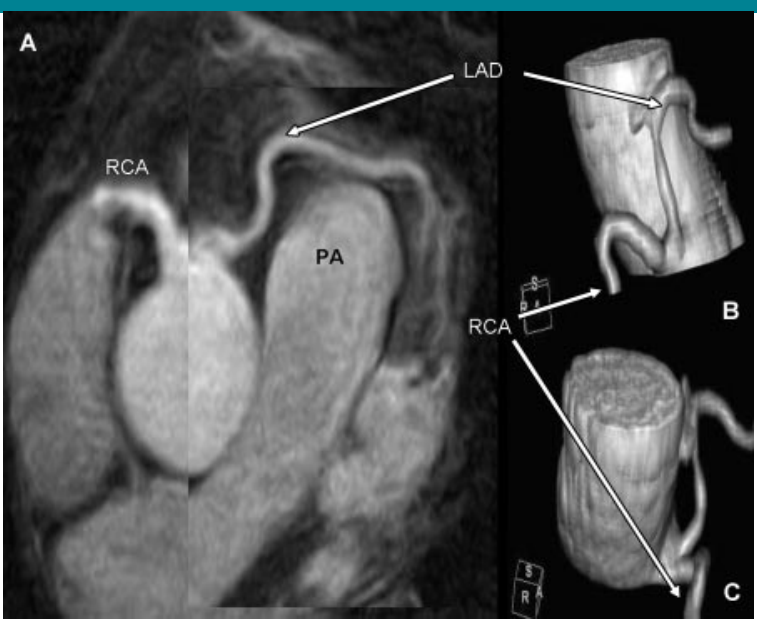

Figure 2: Common origin of the RCA and the LAD in a 62-year-old man with chest pain who experienced diaphoresis and restlessness during coronary MR angiography. To shorten examination time, a 3D navigatorgated segmented gradient-echo sequence (8/2.1, 30 sections, $0.7 \times 1 \times 3$-mm acquired voxel dimension) was performed through the region of the coronary cusps. $A$, Composite image generated by overlaying two reformatted images to create one multiplanar reformatted transaxial image shows the common origin of the $\angle A D$ and the RCA from the right sinus of Valsalva. The LAD passes anteriorly to the pulmonary artery (PA) $B$, Anterior oblique view of the volume-rendered coronary MR angiogram reveals the LAD and the RCA have $a$ common origin. $C$, Right anterior oblique view of the volume-rendered coronary MR angiogram shows the RCA.

tion, which was significant $(P<.001)$. However, there was a tendency to overestimate vessel diameter on MR angiograms, as indicated by the position of the regression line above and to the left of the identity line on the scatterplot (Fig 6). Examination of the regression line shows a slope of approximately 1 . The intercept of $1 \mathrm{~mm}$ offset between the two diameter measurements was not significant $(P=.152)$.

\section{Discussion}

To our knowledge, this is the first study to demonstrate the feasibility of performing free-breathing 3D whole-heart coronary 3-T MR angiography in the clinical assessment of CAAV. A large 3D volume acquisition that included the whole heart was used to successfully depict the coronary arteries in 10 of the 12 patients and in all 10 healthy volunteers. The other two patients were unable to

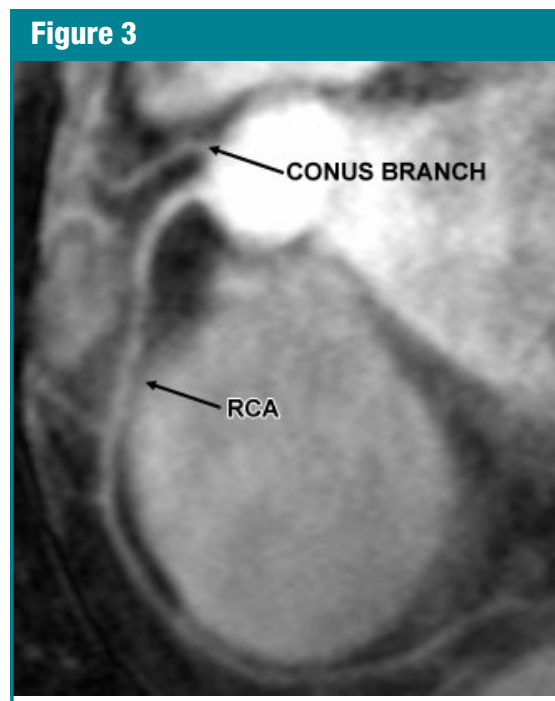

Figure 3: Right anterior oblique multiplanar image reformatted from the whole-heart navigatorgated gradient-echo coronary MR angiogram (4/1.35) obtained in a 30-year-old woman shows a conus branch coronary artery originating from the right sinus of Valsalva instead of from the RCA. 
undergo the longer examination and instead underwent a volume-targeted 3D acquisition. Analogous to CT angiography, which has been shown to be a valuable tool in CAAV evaluation $(37,38)$, whole-heart imaging is preferred because it enables reliable visualization of the entire coronary arterial tree at $3 \mathrm{~T}$ in one acquisition and because it can be used to obtain a $1 \times 1 \times 2$-mm voxel. Additionally, a smaller targeted 3D volume was used to reliably detect the origins of the coronary arteries at the level of the sinuses of Valsalva. This provided a routine in-plane spatial resolution of $0.7 \times 1 \mathrm{~mm}$, which is similar to or slightly better than the spatial resolution previously reported as the best at $1.5 \mathrm{~T}(15-19)$.

Use of higher magnetic field strength increases the signal-to-noise ratio so that it is greater than that at 1.5 T. This principle can be used with $3-\mathrm{T}$ MR to offset the relative signal loss that is inherent in parallel imaging. Thus, the acquisition time can be shortened without substantially compromising image quality (31). The images were obtained in 10-12 minutes with a navigator efficiency of $50 \%-60 \%$. Comparable acquisitions at $1.5 \mathrm{~T}$ generally require a longer examination time (approximately $14-17$ minutes) $(25,27)$. In addition, a shorter imaging time may support improved routine image quality, since navigator efficiency generally deteriorates over time (25). The combination of both large (whole heart) and small 3D volumes resulted in a total imaging time that lasted-on averageapproximately 41 minutes, which is comparable to the imaging time in prior studies (17-19).

Whole-heart 3D free-breathing navigator-gated 1.5-T coronary MR angiography involves the use of steady-state free precession sequences $(25,27,29)$. It is well established that this technique yields a higher signal-to-noise ratio and superior contrast when compared with the more conventional segmented k-space gradient-echo 3-T imaging techniques that were used in an earlier study (39). However, owing to field inhomogene- ities and specific absorption rate restrictions, steady-state free precession sequences cannot easily be used at $3 \mathrm{~T}$ (40). Although there have been developments that have enabled successful implementation of 3-T steady-state free precession sequences (41), they are not yet widely available and may not presently be applicable to large 3D volumes for whole-heart coverage. Thus, the segmented k-space gradient-echo imaging technique is currently preferred for 3-T whole-heart imaging. This may improve further as current manufacturerimposed specific absorption rate limits are more carefully adjusted and steadystate free precession technique details are refined for implementation at $3 \mathrm{~T}$. If the steady-state free precession technique could replace the segmented kspace gradient-echo sequence used in our whole-heart imaging method, a substantial further improvement in vessel conspicuity could be expected. Thus, although a method for acquiring clinically useful coronary MR angiograms at $3 \mathrm{~T}$ has been developed and demonstrated,

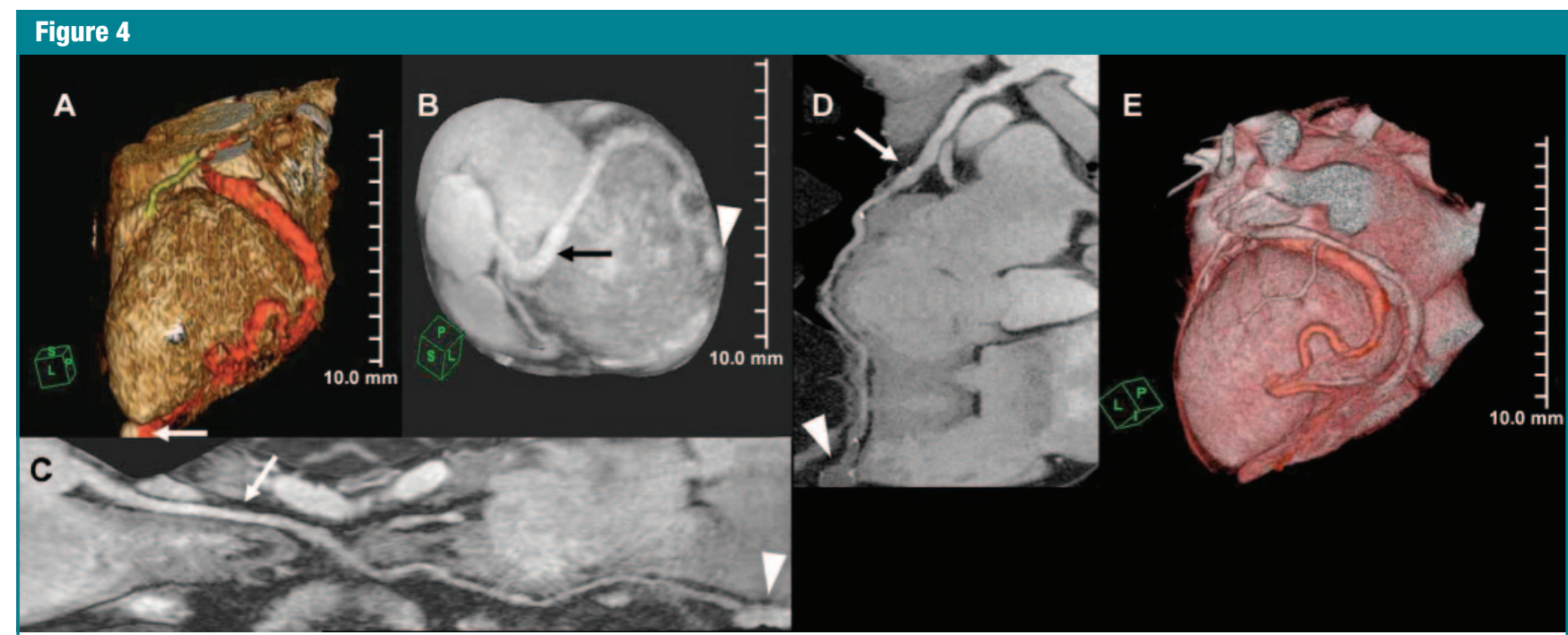

Figure 4: Coronary images obtained in a 47-year-old man who underwent coronary CT angiography because he had had congenital arteriovenous fistula in his childhood. A, Left posterior oblique volume-rendered whole-heart navigator-gated coronary MR angiogram (4/1.35) shows a tortuous and dilated LCX (red) terminating in an aneurysm (arrow) at the tip of the right ventricle. B, A 3D maximum intensity left posterior oblique whole-heart coronary MR angiogram shows the dilated tortuous LCX (arrow) ending in an arteriovenous fistula with the right ventricular apical aneurysm (arrowhead). C, Multiplanar stretch view of the LCX rendered from a whole-heart coronary MR angiogram shows the dilated tortuous LCX (arrow) ending in an arteriovenous fistula with the right ventricular apical aneurysm (arrowhead). D, Multiplanar reformatted stretch view of a contrast-enhanced coronary CT angiogram shows the dilated tortuous LCX (arrow) ending in an arteriovenous fistula at the right ventricular apical aneurysm (arrowhead). This enabled us to confirm what we saw on the MR angiogram. Note the close resemblance of this image to the coronary multiplanar reformatted MR angiogram (Fig 4, C) obtained without contrast enhancement. E, Left posterior oblique volume-rendered contrast-enhanced CT angiogram shows the LCX arteriovenous fistula leading to the right ventricle (red). 


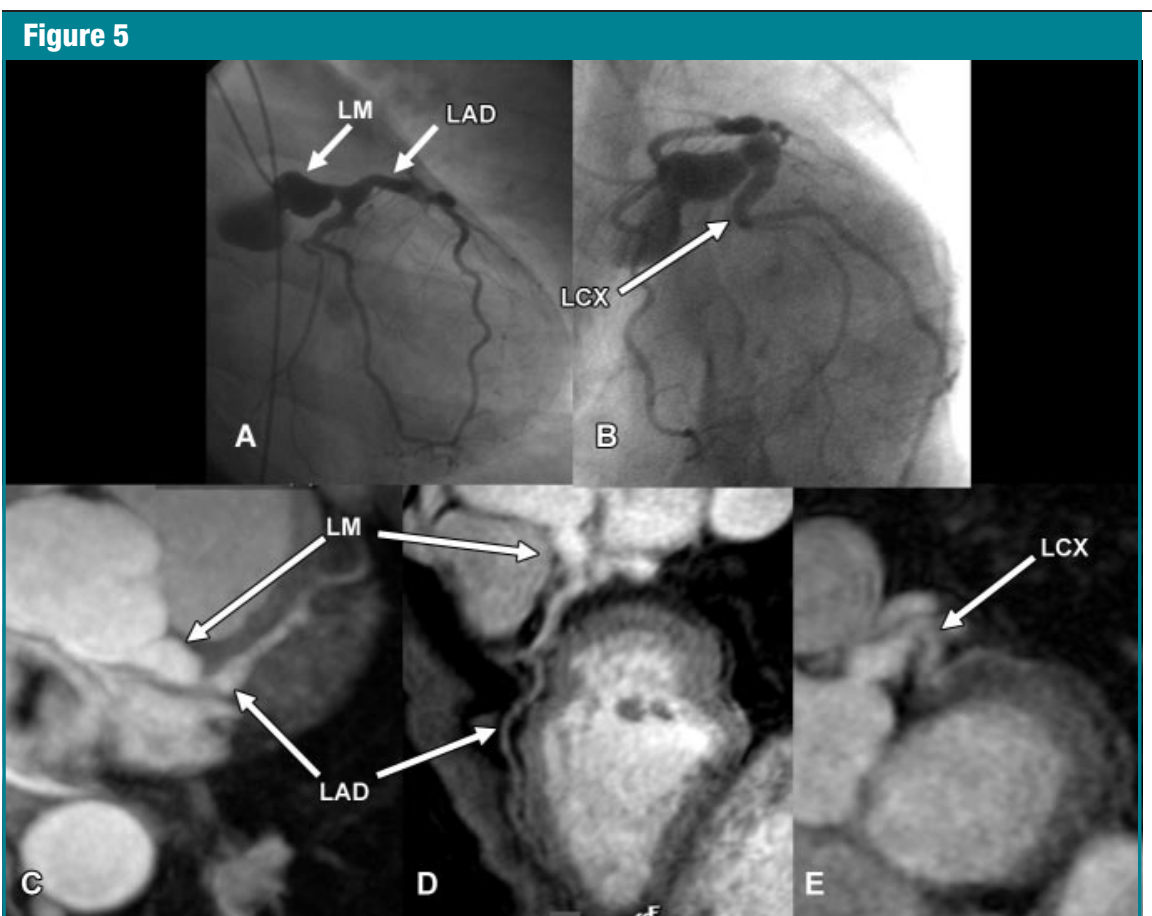

Figure 5: Images obtained in a 48-year-old woman with exertional chest pain. A, Right anterior oblique conventional angiogram shows aneurysmal dilatation of the left main coronary artery (LM)and the proximal LAD. B, Right anterior oblique view obtained with selective injection of the LCX shows angulations involving a short segment of the proximal portion of the LCX. C, Transaxial maximum intensity projection of a segmented gradient-echo navigator-gated whole-heart MR angiogram (4/1.35) shows aneurysmal dilatation of the left main coronary artery (LM) and the proximal LAD. D, Multiplanar stretch image reformatted from the wholeheart MR angiogram shows the entire length of the left main coronary artery $(L M)$ and the $L A D$, as well as the aneurysms seen on the other images. $E$, Multiplanar reformatted image from the whole-heart coronary MR angiogram of the LCX in the right anterior oblique view shows similar angulations noted on the conventional angiogram (Fig 2, $B$ )

it is not yet fully optimized relative to known potential technical avenues for improvement.

We acknowledge the following limitations of our study: This was a small pilot trial in which we did not attempt to examine a large number of patients. However, we were able to show that the technical challenges of $B_{1}$ inhomogeneity and increased specific absorption rate encountered with use of a steadystate free precession sequence at $3 \mathrm{~T}$ could be addressed (with use of segmented gradient-echo sequences, an adiabatic T2 preparation pulse, vector electrocardiographic gating, and volumetric shimming) sufficiently to allow identification of CAAV with 3D wholeheart coronary MR angiography performed at high field strength.

Another limitation was our inability to perform whole-heart acquisition in two patients because they were unable to cooperate. However, in these patients, the more conventional volume-targeted imaging technique enabled us to assign the correct diagnoses, both of which were confirmed with conventional angiography. Finally, it would have been ideal to directly compare images obtained at $1.5 \mathrm{~T}$ with those obtained at $3 \mathrm{~T}$ in the same patients. However, this was impossible owing to practical limitations. Furthermore, it was not the goal of this feasibility study. Now that we have demonstrated the feasibility of using this technique with 3-T MR angiography to reliably evaluate CAAV, a direct head-to-head comparison with 1.5-T MR angiography can be performed. However, the challenges of different techniques (the steady-state

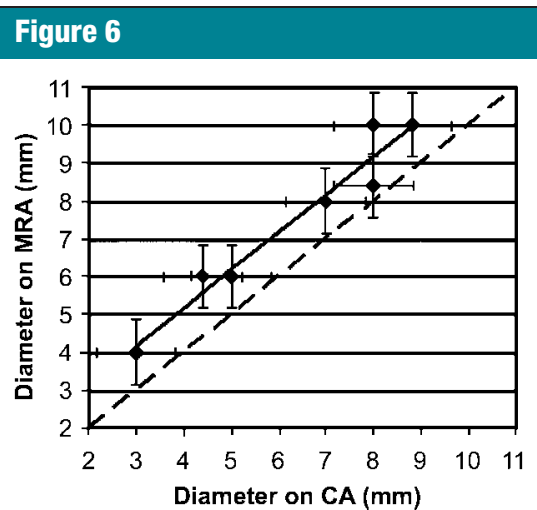

Figure 6: Scatterplot shows the relationship between conventional angiography $(C A)$ and $M R$ angiography (MRA) for ectatic and aneurysmal coronary vessel diameter measurements. The data points (diamonds with error bars) and the regression line (solid line) are to the left of the identity line (dashed line), signifying overestimation of diameter measurements with MR angiography. The following equation was used to plot the regression line: $y=1.1664+1.0008 x$. Pvalues for the intercept and the slope, respectively, are .152 and $<.001\left(R^{2}=0.95\right)$

free precession technique vs the segmented gradient-echo technique), the availability of both 1.5-T and 3-T systems equipped with the same hardware and software, and the scheduling and/or accessibility constraints will need to be resolved before a meaningful comparison can be made.

In summary, our findings demonstrate the usefulness of this method, which was specifically adapted to evaluate a variety of CAAV with 3-T MR angiography. This technique was not only useful in the identification of anomalous coronary origins but also capable of being used to identify and measure the dimensions of aneurysms. This is consistent with prior 1.5-T MR findings (42). However, Greil et al (42) described a tendency to mildly overestimate luminal diameter on 1.5-T MR angiograms obtained in patients with $\mathrm{Ka}$ wasaki disease. This trend may be due to partial volume effects from the relatively large voxel sizes at $1.5 \mathrm{~T}$. Further technical improvements at $3 \mathrm{~T}$ that are centered on refining the steady-state free precession sequence to permit broader use are anticipated and may yield images of even better quality. 


\section{References}

1. Angelini P, Velasco JA, Flamm S. Coronary anomalies: incidence, pathophysiology, and clinical relevance. Circulation 2002;105:2449-2454.

2. Varghese A, Keegan J, Pennell DJ. Cardiovascular magnetic resonance of anomalous coronary arteries. Coron Artery Dis 2005;16:355-364.

3. Baltaxe HA, Wixson D. The incidence of congenital anomalies of the coronary arteries in the adult population. Radiology 1977;122:47-52.

4. Click RL, Holmes DR Jr, Vlietstra RE, Kosinski AS, Kronmal RA. Anomalous coronary arteries: location, degree of atherosclerosis, and effect on survival-a report from the Coronary Artery Surgery Study. J Am Coll Cardiol 1989;13:531-537.

5. Yamanaka O, Hobbs RE. Coronary artery anomalies in 126,595 patients undergoing coronary arteriography. Cathet Cardiovasc Diagn 1990;21: $28-40$.

6. Alexander RW, Griffith GC. Anomalies of the coronary arteries and their clinical significance. Circulation $1956 ; 14: 800-805$

7. Welker M, Salanitri J, Deshpande VS, Shea SM, Li D, Pereles FS. Coronary artery anomalies diagnosed by magnetic resonance angiography. Australas Radiol 2006;50:114-121.

8. Le T, Laskey WK, McLaughin J, White C. Utility of magnetic resonance imaging in a patient with anomalous origin of the right coronary artery, acute myocardial infarction, and near-sudden cardiac death. Cathet Cardiovasc Diagn 1997;42: 205-207.

9. Cox ID, Bunce N, Fluck DS. Failed sudden cardiac death in a patient with an anomalous origin of the right coronary artery. Circulation 2000;102: 1461-1462.

10. Maron BJ, Thompson PD, Puffer JC, et al. Cardiovascular preparticipation screening of competitive athletes: a statement for health professionals from the Sudden Death Committee (clinical cardiology) and Congenital Cardiac Defects Committee (cardiovascular disease in the young), American Heart Association. Circulation 1996;94:850-856.

11. Roberts WC, Siegel RJ, Zipes DP. Origin of the right coronary artery from the left sinus of valsalva and its functional consequences: analysis of 10 necropsy patients. Am J Cardiol 1982;49:863868.

12. Liberthson RR, Dinsmore RE, Fallon JT. Aberrant coronary artery origin from the aorta: report of 18 patients, review of literature, and delineation of natural history and management. Circulation 1979;59:748-754.

13. Cheitlin MD, De Castro CM, McAllister HA. Sudden death as a complication of anomalous left coronary origin from the anterior sinus of valsalva, a not-so-minor congenital anomaly. Circulation $1974 ; 50: 780-787$

14. Yucel EK, Anderson CM, Edelman RR, et al. AHA scientific statement: magnetic resonance angiography-update on applications for extracranial arteries. Circulation 1999;100:2284-2301.

15. Taylor AM, Thorne SA, Rubens MB, et al. Coronary artery imaging in grown up congenital heart disease: complementary role of magnetic resonance and x-ray coronary angiography. Circulation 2000:101:16701678

16. Vliegen HW, Doornbos J, de Roos A, Jukema JW, Bekedam MA, van der Wall EE. Value of fast gradient echo magnetic resonance angiography as an adjunct to coronary arteriography in detecting and confirming the course of clinically significant coronary artery anomalies. Am J Cardiol 1997; 79:773-776.

17. McConnell MV, Ganz P, Selwyn AP, Li W, Edelman RR, Manning WJ. Identification of anomalous coronary arteries and their anatomic course by magnetic resonance coronary angiography. Circulation 1995; 92:3158-3162.

18. Bunce NH, Lorenz CH, Keegan J, et al. Coronary artery anomalies: assessment with free-breathing three-dimensional coronary MR angiography. Radiology 2003;227:201-208.

19. Post JC, van Rossum AC, Bronzwaer JG, et al. Magnetic resonance angiography of anomalous coronary arteries: a new gold standard for delineating the proximal course? Circulation 1995;92:3163-3171.

20. Casolo G, Del Meglio J, Rega L, et al. Detection and assessment of coronary artery anomalies by three-dimensional magnetic resonance coronary angiography. Int J Cardiol 2005;103:317.

21. Pruessmann KP, Weiger M, Scheidegger MB, Boesiger P. SENSE: sensitivity encoding for fast MRI. Magn Reson Med 1999;42:952-962.

22. Fischer SE, Wickline SA, Lorenz CH. Novel realtime R-wave detection algorithm based on the vectorcardiogram for accurate gated magnetic resonance acquisitions. Magn Reson Med 1999; 42:361-370.

23. Stuber M, Botnar RM, Fischer SE, et al. Preliminary report on in vivo coronary MRA at 3 Tesla in humans. Magn Reson Med 2002;48:425-429.

24. Botnar RM, Stuber M, Danias PG, Kissinger KV, Manning WJ. Improved coronary artery definition with T2-weighted, free-breathing, three-dimensional coronary MRA. Circulation 1999;99:31393148 .

25. Jahnke C, Paetsch I, Nehrke K, et al. Rapid and complete coronary arterial tree visualization with magnetic resonance imaging: feasibility and diagnostic performance. Eur Heart J 2005;26:23132319.

26. Kim WY, Danias PG, Stuber M, et al. Coronary magnetic resonance angiography for the detection of coronary stenoses. N Engl J Med 2001;345: 1863-1869.

27. Sakuma H, Ichikawa Y, Suzawa N, et al. Assessment of coronary arteries with total study time of less than 30 minutes by using whole-heart coronary MR angiography. Radiology 2005;237:316321.

28. Stuber M, Botnar RM, Danias PG, et al. Doubleoblique free-breathing high resolution three-dimensional coronary magnetic resonance angiography. J Am Coll Cardiol 1999;34:524-531.

29. Weber OM, Martin AJ, Higgins CB. Whole-heart steady-state free precession coronary artery mag- netic resonance angiography. Magn Reson Med 2003;50:1223-1228.

30. Sommer T, Hackenbroch M, Hofer U, et al. Coronary MR angiography at $3.0 \mathrm{~T}$ versus that at $1.5 \mathrm{~T}$ : initial results in patients suspected of having coronary artery disease. Radiology 2005;234:718 725 .

31. Huber ME, Kozerke S, Pruessmann KP, Smink J, Boesiger P. Sensitivity-encoded coronary MRA at 3T. Magn Reson Med 2004;52:221-227.

32. Brittain JH, Hu BS, Wright GA, Meyer CH, Macovski A, Nishimura DG. Coronary angiography with magnetization-prepared T2 contrast. Magn Reson Med 1995;33:689-696.

33. Nezafat R, Stuber M, Ouwerkerk R, Gharib AM, Desai MY, Pettigrew RI. B1-insensitive T2 preparation for improved coronary magnetic resonance angiography at 3 T. Magn Reson Med 2006;55: $858-864$

34. Stuber M, Botnar RM, Danias PG, Kissinger KV, Manning WJ. Submillimeter three-dimensional coronary MR angiography with real-time navigator correction: comparison of navigator locations. Radiology 1999;212:579-587.

35. Etienne A, Botnar RM, Van Muiswinkel AM, Boesiger P, Manning WJ, Stuber M. "Soap-Bubble" visualization and quantitative analysis of 3D coronary magnetic resonance angiograms. Magn Reson Med 2002;48:658-666.

36. Grimbacher B, Holland SM, Gallin JI, et al. HyperIgE syndrome with recurrent infections: an autosomal dominant multisystem disorder. N Engl J Med 1999;340:692-702.

37. Datta J, White CS, Gilkeson RC, et al. Anomalous coronary arteries in adults: depiction at multidetector row CT angiography. Radiology 2005; 235:812-818.

38. van Ooijen PM, Dorgelo J, Zijlstra F, Oudkerk M. Detection, visualization, and evaluation of anomalous coronary anatomy on 16 slice multidetector-row CT. Eur Radiol 2004; 14 : 2163-2171.

39. Spuentrup E, Buecker A, Stuber M, et al. Navigator-gated coronary magnetic resonance angiography using steady-state-free-precession: compari son to standard T2-prepared gradient-echo and spiral imaging. Invest Radiol 2003;38:263-268.

40. Kaul MG, Stork A, Bansmann PM, et al. Evalua tion of balanced steady-state free precession (TrueFISP) and K-space segmented gradient echo sequences for 3D coronary MR angiography with navigator gating at 3 Tesla. Rofo 2004;176:1560 1565 .

41. Schar M, Kozerke S, Fischer SE, Boesiger P. Cardiac SSFP imaging at 3 Tesla. Magn Reson Med 2004;51:799-806.

42. Greil GF, Stuber M, Botnar RM, et al. Coronary magnetic resonance angiography in adolescents and young adults with Kawasaki disease. Circulation 2002;105:908-911. 